\title{
Human Errors - A Taxonomy for Describing Human Malfunction in Industrial Installations
}

Rasmussen, Jens

Published in:

Journal of Occupational Accidents

Link to article, DOI:

10.1016/0376-6349(82)90041-4

Publication date:

1982

Document Version

Peer reviewed version

Link back to DTU Orbit

Citation (APA):

Rasmussen, J. (1982). Human Errors - A Taxonomy for Describing Human Malfunction in Industrial Installations. Journal of Occupational Accidents, 4(2-4), 311-333. https://doi.org/10.1016/0376-6349(82)90041-4

\section{General rights}

Copyright and moral rights for the publications made accessible in the public portal are retained by the authors and/or other copyright owners and it is a condition of accessing publications that users recognise and abide by the legal requirements associated with these rights.

- Users may download and print one copy of any publication from the public portal for the purpose of private study or research.

- You may not further distribute the material or use it for any profit-making activity or commercial gain

- You may freely distribute the URL identifying the publication in the public portal

If you believe that this document breaches copyright please contact us providing details, and we will remove access to the work immediately and investigate your claim 


\title{
Human Errors: A Taxonomy for Describing Human Malfunction in Industrial Installations ${ }^{1}$
}

\author{
Jens Rasmussen
}

\begin{abstract}
This paper describes the definition and the characteristics of human error. Different types of human behavior are classified, and their relation to different error mechanisms are analyzed. The effect of conditioning factors related to affective, motivating aspects of the work situation as well as physiological factors are also taken into consideration. The taxonomy for event analysis, including human malfunction, is presented. Possibilities for the prediction of human error are discussed. The need for careful studies in actual work situations is expressed. Such studies could provide a better understanding of the complexity of human error situations as well as the data needed to characterize these situations.
\end{abstract}

\section{INTRODUCTION}

At present we witness a large interest in the involvement of human operators in the reliability and safety of industrial installations as well as in methods for incorporation of the effects of human errors in quantitative risk assessment. Two features of the present situation are particularly important when considering the development of quantitative methods: One is the need to consider the human role in rare events due to the risk involved in large scale industrial installations. Another one is the introduction of interface equipment based on the rapidly developing information technology. Together these features lead to a pronounced need for replacement of empirical design guides by tools and methods for analytical human performance prediction and error probability estimation.

Analytical techniques are based on a causal model of the role of parts and components of a system in the accidental chains of events leading from the initial fault or disturbance to the unacceptable consequences. Until recently, most analytical effort for including human errors has been spent on verification of the safety of existing, operating process plants of traditional design with respect to the man-machine interface, as for instance, the use of Therp (Swain 1976) on nuclear power plants (WASH 1400). This situation has led to a definition of errors and quantification of error rates in terms referring to the structure and elements of the external human task, and not in terms of human functions and capabilities and their limitations. Generally, current methods are based on a taxonomy of human errors in terms of erro-

\footnotetext{
${ }^{1}$ Journal of Occupational Accidents, Vol. 4, Nos. 2-4 pp. 311-333, 1982
} 
neous sequencing or performance of task elements or steps; i.e., the analytical approach is based on a model of the task rather than a model of the man performing the task. Human functions and features are then taken into consideration by means of "performance shaping factors" (Swain 1976, 1980).

Unfortunately this means that only little guidance for predicting human performance with new designs of man-machine interfaces can be derived. To be able to collect empirical data for human performance for transfer to another task context than the one supplying the data, a generic psychological classification of human errors must be applied which has well specified relations to generic task properties and environmental features.

Only little is published on such generic psychological error mechanisms, probably because human errors have been considered to be a weakness of operators which could be cured by improved training and better instructions and because the pace of change of work situations has been slow enough to allow for purely empirical methods. Typically, some of the early attempts to find generic psychological error mechanisms from analysis of professional task performance are from aviation research to improve cockpit designs see for instance Fitts and Jones (1947). Theoretical studies of psychological error mechanisms have only started quite recently. James Reason $(1975,76,79)$ has published analysis of human errors based on explicit models of human performance and has made attempts to sketch a taxonomy of error mechanisms from the analysis of "every day slips and lapses".

Based on analysis of event reports from nuclear power plants an attempt has been made to characterize human error mechanisms in generic terms (Rasmussen 1980) and the results of this analysis have been adopted in a proposal for a taxonomy for practical data collection by an OECD/CSNI group of experts (Rasmussen et al. 1981). Recently, also Norman $(1979,80)$ has published analysis of every day "slips of mind" including initial attempts to develop more generic classification schemes for description of human errors. However, before discussing a taxonomy of human errors, it will be necessary to consider a definition of human errors in more detail.

\section{DEFINITION AND CHARACTERISTICS OF HUMAN ERRORS}

Basically it is very difficult to give a satisfactory definition of human errors. Frequently they are identified after the fact: If a system performs less satisfactorily than it normally does due to a human act or to a disturbance which could have been counteracted by a reasonable human act the cause will very likely be identified as a human error. This is 
probably because the analyst will not have the information or psychological background which is necessary to trace through the human performance in the explanatory causal backtracking process to find a possible causal input (see Rasmussen 1980).

A more fruitful point of view is to consider human errors as instances of man-machine or man-task misfits. In case of systematic or frequent misfits, the cause will typically be considered to be a design error. Occasional misfits are typically caused by variability on part of the system or the man and are considered to be system failures or human errors, respectively.

However, human variability is an important ingredient in adaptation and learning and the ability to adapt to peculiarities in system performance and optimize interaction is the very reason for having people in a system. To optimize performance, to develop smooth and efficient skills, it is very important to have opportunities to perform trial and error experiments, and human errors can in a way be considered as unsuccessful experiments with unacceptable consequences. Typically they are only classified as human errors because they are performed in an "unkind" work environment. An unkind work environment is then defined by the fact that it is not possible for a man to observe and reverse the effects of inappropriate variations in performance before they lead to unacceptable consequences.

When the effect of human variability is observable and reversible, the definition of error is related to a reference or norm in terms of the successful outcome of the activity. However, if observability and/or reversibility of inappropriate performance are not present; if, for instance, the effect of errors is delayed in time, is depending on further steps in a sequence, or is dependent upon possible latent conditions, as is often the case in industrial installations, then an established successful procedure becomes the man's only immediate reference for a judgment of errors, which therefore are related to the activity per se, rather than to a fulfillment of the related goal.

In practice it will be very difficult to arrange data collection related to those human errors which are immediately observable and reversible by the acting person and, therefore, may be corrected without further notice. In particular, it will be very difficult to determine the frequency of opportunities for such errors which will be needed to derive error rates or probabilities of error in a specific situation.

The features of observability and reversibility vary with error types and with task context and depend on very specific and detailed characteristics of the interface. Consequently, different task settings will be potential sources of data for the various error types. In order to transfer error data to predict performance in a new task design, it is necessary 
to have a match between observability and reversibility features of the new task and those used as data sources for each of the various relevant error mechanisms. It is, therefore, necessary to identify and characterize the features of a task which prevent error reversal during the analyzed events and which, during data collection, will act as a selective filter upon the initial repertoire of errors committed. This should probably not be part of the event analysis itself but be performed as a background task analysis for the work situations included in the data collection.

The analytical problems depend very much on the nature of the work situation. In process plant control, for instance, the work situation is highly structured and the effects of human errors can be analyzed. However, due to the processes which are not immediately visible, the recovery problem is rather complex. In general work safety, the work situation is very unstructured, which causes analytical problems; on the other hand, the "processes" are typically rather concrete and visible, and recovery features can be more readily defined.

\section{HUMAN PERFORMANCE AND ERROR MECHANISMS}

By analytical systems assessment, the consequences and probabilities of accidental chains of events are predicted from a causal model based on knowledge of failure modes of the components of the system and the related failure rates. To facilitate systematic analysis, a library of "minifault-trees" for standard components is generally used. Probabilities for the failure modes related to different branches of the tree are typically conditioned by operating and environmental parameters. The basic idea is that the components can be represented by an input-output model with known transfer functions for the various failure modes. Similarly, human system "components" have been represented by input-output models with transfer characteristics which can be modified by error mechanisms (see fig. 1).

Already from the above discussion of the definition of human errors, it appears that this model is unrealistic since it does not take into consideration the selective filtering of error mechanisms depending upon reversibility features of the task context. Furthermore, the model lacks the aspect of human intention and expectancy; in reality, there is no one-to-one relationship between the external task performance and the internal human functions which are used. This relationship depends on human value perception and upon subjective goals and performance criteria derived from such values. From the subjective goals and the expectations about the state of the system, information is sought and collected actively. Trained people ask questions to the system, biased by 
their experience and immediate expectations; they do not passively receive and filter an information input (see fig. 2).

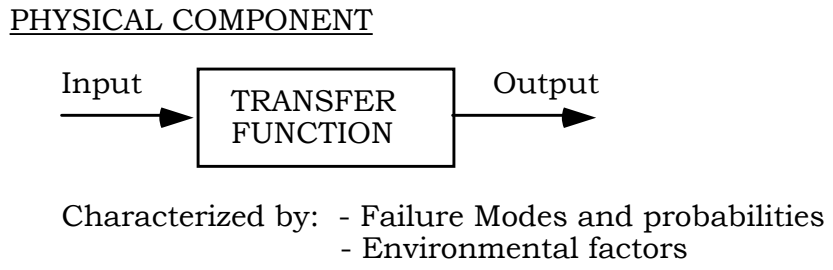

- Environmental factors

HUMAN OPERATOR

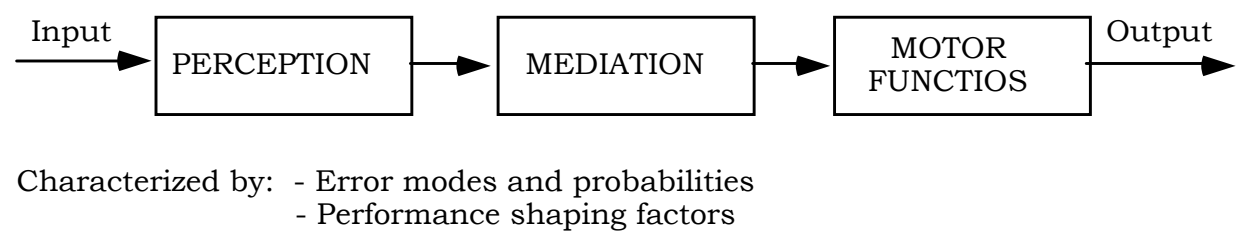

Figure 1. Schematic diagram illustrating input-output response model of a physical component used for failure analysis and the analog model frequently adopted for human operators.

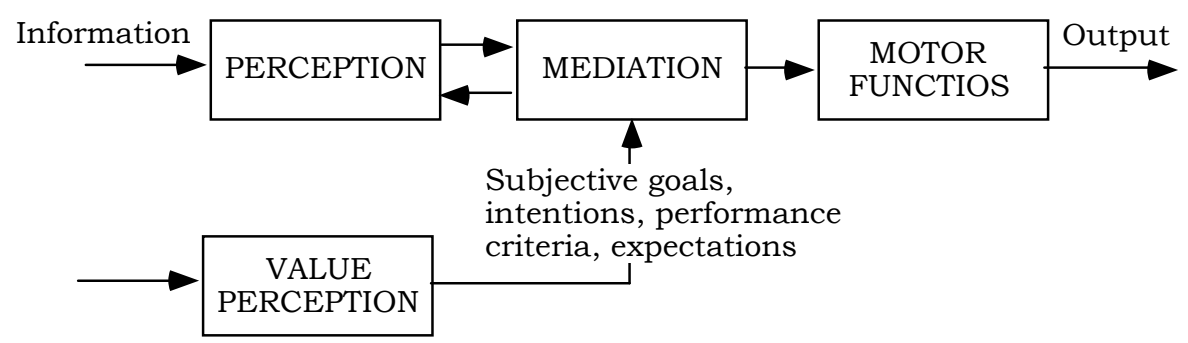

Figure 2. Model of a human operator must include active intentions, expectations, and subjective goals.

It follows that error mechanisms and failure modes depend on mental functions and knowledge which are activated by subjective factors. They cannot be directly observed but must be inferred from characteristics of the task and the work situation together with the external manifestation of the error. For this to be possible, a model of human information processing must be available. Such a model must relate elements of human decision making and action to internal information processes for which generic psychological mechanisms and limitations can be identified. An attempt to develop such a model from analysis of verbal protocols and cases of human malfunction has been described elsewhere (Rasmussen 1976, 80).

In this model, a distinction is drawn between three levels of behavior: skill-, rule-, and knowledge-based performance, see fig. 3. This distinction is tightly related to the norm or reference used for error judgment, since different concepts are used to control behavior: In the skill-based 
domain, including automated, more or less subconscious routines, performance is controlled by stored patterns of behavior in a time-space domain. Errors are related to variability of force, space or time coordination. The rule-based domain includes performance in familiar situations controlled by stored rules for coordination of subroutines, and errors are typically related to mechanisms like wrong classification or recognition of situations, erroneous associations to tasks, or to memory slips in recall of procedures. Since rule-based behavior is used to control skill-based subroutines, the error mechanisms related to skillbased routines are always active. Rule-based behavior is not directly goal-controlled, but goal oriented, and the immediate criteria for errors deal with whether the relevant rules are recalled and followed correctly or not. This is the case, unless the total task is considered explicitly as one integrated whole and ultimate error correction is included in the error definition. However, in that case, transfer of data to and from a different task context is not possible.

The third behavioral domain is called upon in case of unique, unfamiliar situations for which actions must be planned from an analysis and decision based on knowledge of the functional, physical properties of the system and the priority of the various goals. In this domain, the internal data processing functions used for the task are very person and situation dependent and vary with details in the task context, with the extent and type of knowledge immediately available to the person, and with his subjective preferences. In general, errors in this domain can only be defined in relation to the goal of the task and generic error mechanisms can only be defined from very detailed studies based on verbal protocols which can supply data on the actual data process.

Data collection and prediction based on a breakdown of task performance in the knowledge-based domain is only possible for very tightly controlled experimental situations, not for real-life task settings (Rasmussen 1980). Consequently, the present taxonomy only includes inappropriate reading of input information together with errors of inference leading to unsuccessful performance due to latent, not considered, conditions or to unacceptable side effects. Both are failures in functional reasoning about a causal network. 


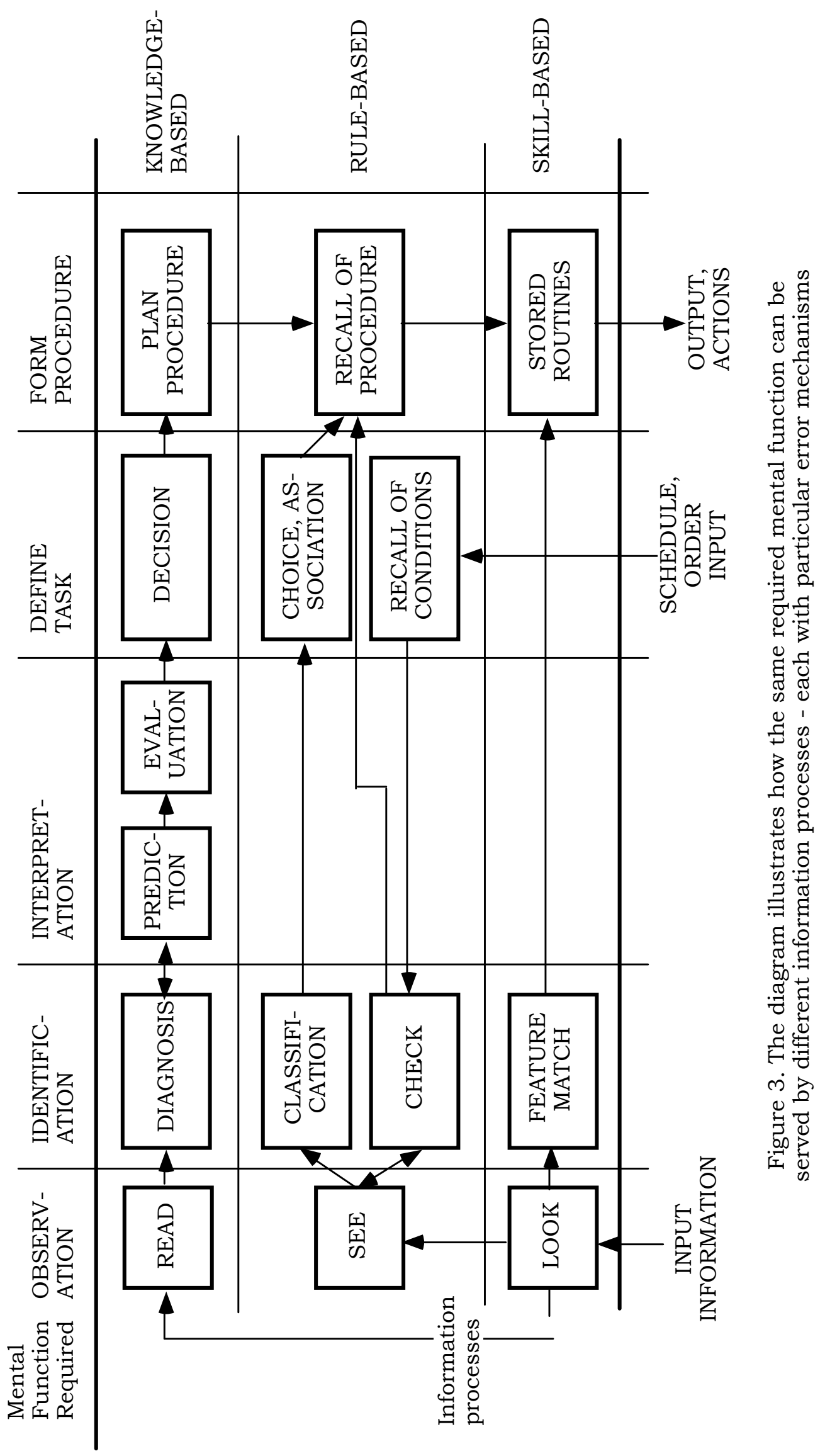


An important set of error mechanisms is related to failure in selecting the proper level of behavior in an abnormal situation, here called errors of discrimination. These error mechanisms are consequences of the fact that data in the environment cannot be considered input information to a passive data processor. In the three levels of behavior, a man uses basically different information which is derived from the data, viz. information in the form of signals, signs, or symbols (Rasmussen 1980). Which interpretation he uses depends on an active choice and error mechanisms are related to his bias or fixation for this choice.

Fig. 3 illustrates the characteristic data processes of the three levels. Clearly, the mental processes and the related error mechanisms are different for the various levels. The level applied in a given situation depends strongly upon the degree of training of the operator, and it is seen that error data collected from routine task situations are not applicable in unfamiliar, infrequent situations (such as emergencies) irrespective of the effects of stress and similar factors. In passing it can be mentioned that "tunnel vision" during emergency situations can be the effect not only of stress, but can be caused by the fundamental nature of the diagnostic task (cognitive tunneling, Moray 1981) and of the capacity requirements of higher level mental tasks (Rasmussen 1981).

The generic mental functions at the three levels of behavior as illustrated in the schematic diagram of fig. 3 must be related to a more general description of that internal mental function which was not performed as required by the external task. In order to be able to identify the internal function which failed on the basis of the external effects of errors alone, this description must be independent on the level of human behavior and based alone on a rational breakdown of the decision sequence into the phases of detection, identification, decision, etc., as indicated on top of fig. 3 .

So far we have considered only what went wrong (the internal mental function that failed) and how it went wrong (the internal failure mechanism) together with the effect upon the external task, the external mode of malfunction. It is necessary also to consider explicitly the cause of the malfunction, why it happened, especially whether the change in the work is ascribed the role of cause, is related to spontaneous human variability or is a change in the external work condition, in the form of a change in task content or an irrelevant, distracting event. In conclusion, these factors add up to a description of a human error in the form of a causal chain of events as shown in fig. 4.

How far back in this chain one needs to go to identify the category to be selected as the nominal cause for which data is collected depends upon the actual use. To judge reliability of an existing task, it is only necessary to consider the external mode of failure; to judge training and 
interface design for improvement, the mechanism of failure must be considered; and to evaluate the work situation, the external causes must also be identified.

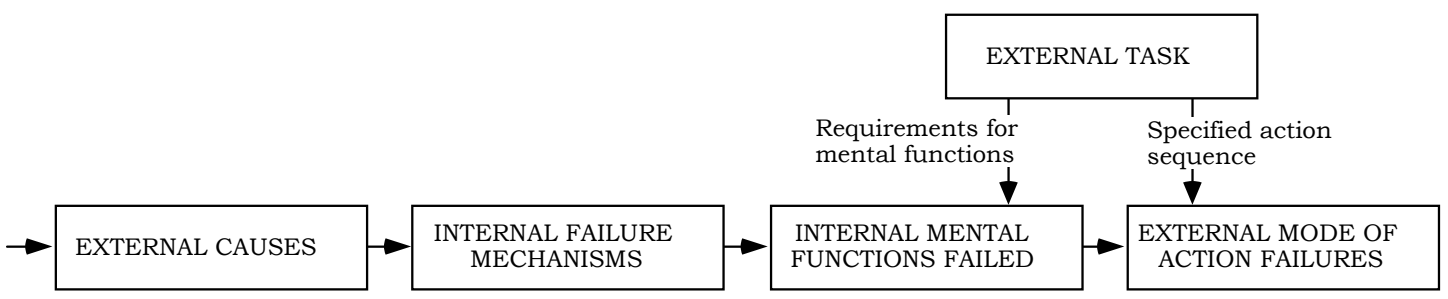

Figure 4. Typical characteristics of an accidental chain of events including human malfunction.

Fig. 4 include five aspects of human errors which are useful as five dimensions in a multi-facet classification system. These dimensions are not completely independent, significant correlation is found for subsets of tasks and work situations. Furthermore, the members of the different categories are not mutually exclusive and, therefore, generic fault trees or prototypical decision-error-trees are not feasible to characterize human performance. We prefer the use of a multi-facet description from which error-trees can be derived for specific applications, as described later. This solution also seems to be preferable for computer administration and analysis of data since a good resolution can be obtained with a limited number of classes in each dimension.

\section{PERFORMANCE SHAPING FACTORS}

The causal chain of events of fig. 4 only considers the information processing aspects of the man-machine interaction; i.e., the chain of events related to changes in the conditions for human decisions or to the process of decision making itself. However, the work environment influences man in a much more complex way than through the information domain alone, as illustrated by fig. 5 . It appears to be necessary to include conditioning factors related to affective, motivating aspects of the work situation as well as physiological factors. Such factors will not directly appear in the causal chain of events but may influence it by changing human limits of capability, subjective preferences in choice of mental strategies and goals etc. Some of these factors can only be identified by careful analysis of the actual work "climate" and are frequently not considered in normal event reports. Together with the categories of fig. 5, such conditioning factors result in the taxonomy of human errors illustrated by fig. 6 . 
INFLUENCE FROM

ENVIRONMENT

\section{HUMAN OPERATOR FUNCTIO}

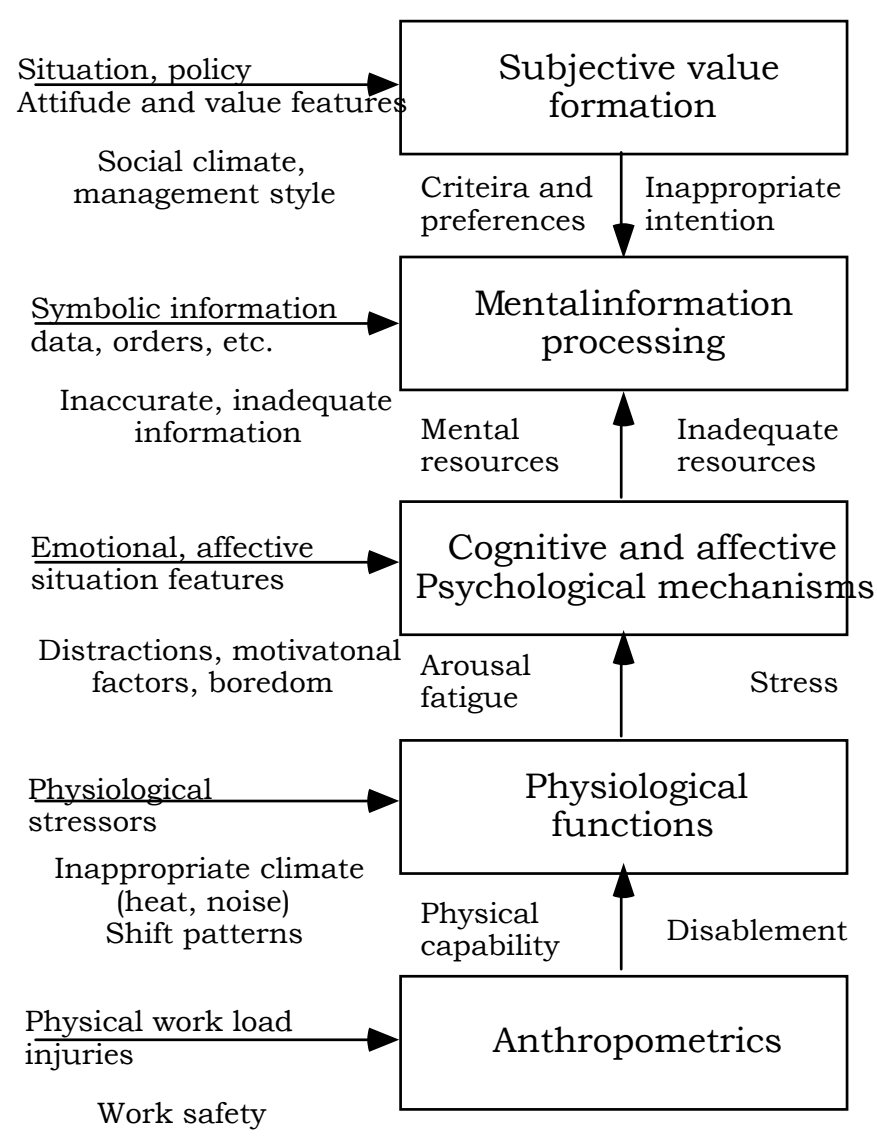

Figure 5 illustrates the complex interactions in a man-machine system which control the mis-match found in an error situation.

\section{THE TAXONOMY FOR EVENT ANALYSIS INCLUDING HUMAN MALFUNCTION}

The categories of the taxonomy directly related to the inappropriate human performance are shown on fig. 6 . When used for data collection in process plant environments, a number of categories are added for description of the circumstances for the event, including characteristics of the process plant and its immediate operational state; the manner of detection of the event; the ultimate consequences upon plant operation; the systems and components affected; the personnel category involved; the work location; etc. These categories are discussed in more detail in Rasmussen et al. 1981.

When used for data collection, a number of conventions are needed to avoid too much ambiguity and guidelines for event analysis should be used for consistent classification. Guidelines are proposed elsewhere (Hollnagel et al. 1981) and examples are given in the following discussion of the categories, which is ordered according to the logical se- 
quence of analysis. It will be clear from the discussion that the important feature of the taxonomy proposed will be the structure and its dimensions, not the detailed numbers of categories, which may vary with the specific application.

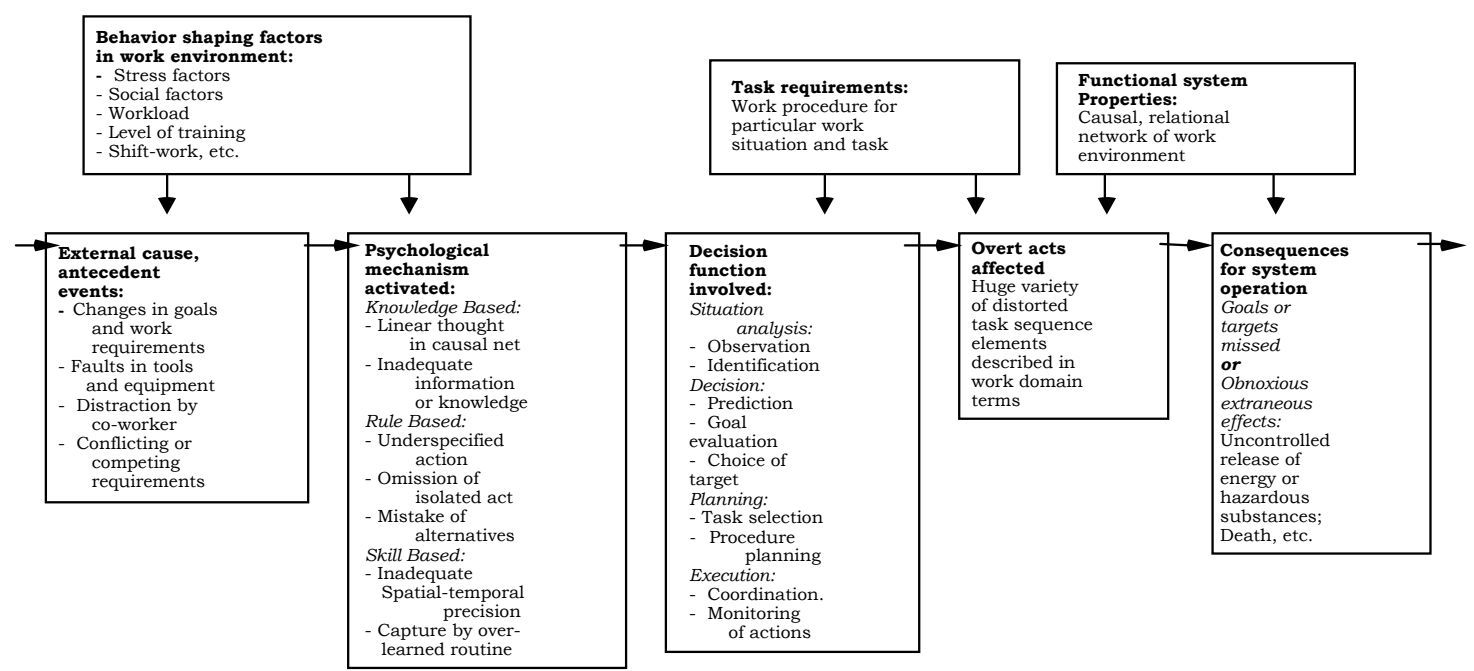

Figure 6. Multi-facet taxonomy for description and analysis of events involving human malfunction

\section{Personnel Task}

Identification of the task performed is important to characterize the circumstances during which the malfunction occurred. The categories included in fig. 7 are rather general and only useful for routine event reporting. In proper data collection campaigns, the tasks involved must be analyzed and the location of the failure in the task more precisely identified. Furthermore, task analysis must be performed to determine the bias resulting from the potential for immediate error correction together with the frequency of error opportunities.

\section{External Mode of Malfunction}

This category describes the immediate, observable effect of human malfunction upon the task performance and the way in which it initiates the consequent chain of accidental events. The category serves to characterize the sensitivity of the system to the malfunction in a few classes which are useful for monitoring a plant system by routine reports and which relates to the information needed in reliability analysis (whether the specified task is performed) and for risk analysis (effects of erroneous acts). For predictive analysis, the classes are not very useful and more specific external modes should be determined from a correlation of error mechanisms, internal functions and the result of a task analysis as described below. 
Internal Human Malfunction

This category identifies the internal mental function of the man's decision making which was not performed as required by the task. It is based upon a model of human decision making as a rational sequence of elements as indicated in fig. 3, which may be performed as stated or by-passed by habitual leaps. Event analysis should serve to identify the decision process that has been erroneously performed or has been inappropriately by-passed by a habitual leap.

The use of these decision categories is ambiguous in several ways and some conventions are necessary to give consistent classifications. First of all, human performance has basically a hierarchical-structure and it may consequently be a matter of choice as to which level the decision categories are used and how they are brought into use. This choice will depend on the circumstances during which inappropriate human performance is found and on the amount and quality of the information available from the event. One typical example will be a skilled operator making a single erroneous decision during normal or near-normal work situations. In this case, the decision categories will be used on a high level of task planning, partly because highly professional people are only making "decisions" at a high level to control their skilled and more subconscious routines as illustrated in fig. 3, and partly because routine event reports do not include information which enables an identification of decision errors at a lower level, even though they may appear; e.g., if a skilled routine must be modified. A repair task can be taken as an example: If the equipment fault is incorrectly diagnosed, the inappropriate mental function is classified as "identification". However, if the fault is correctly identified and the task of replacement properly mentioned but inappropriately planned because the internal state of the equipment is not properly identified at a lower level, then the mental malfunction will be classified as "inappropriate procedure". 
ERRONEOUS DECISION FUNCTION: WHAT FAILED?

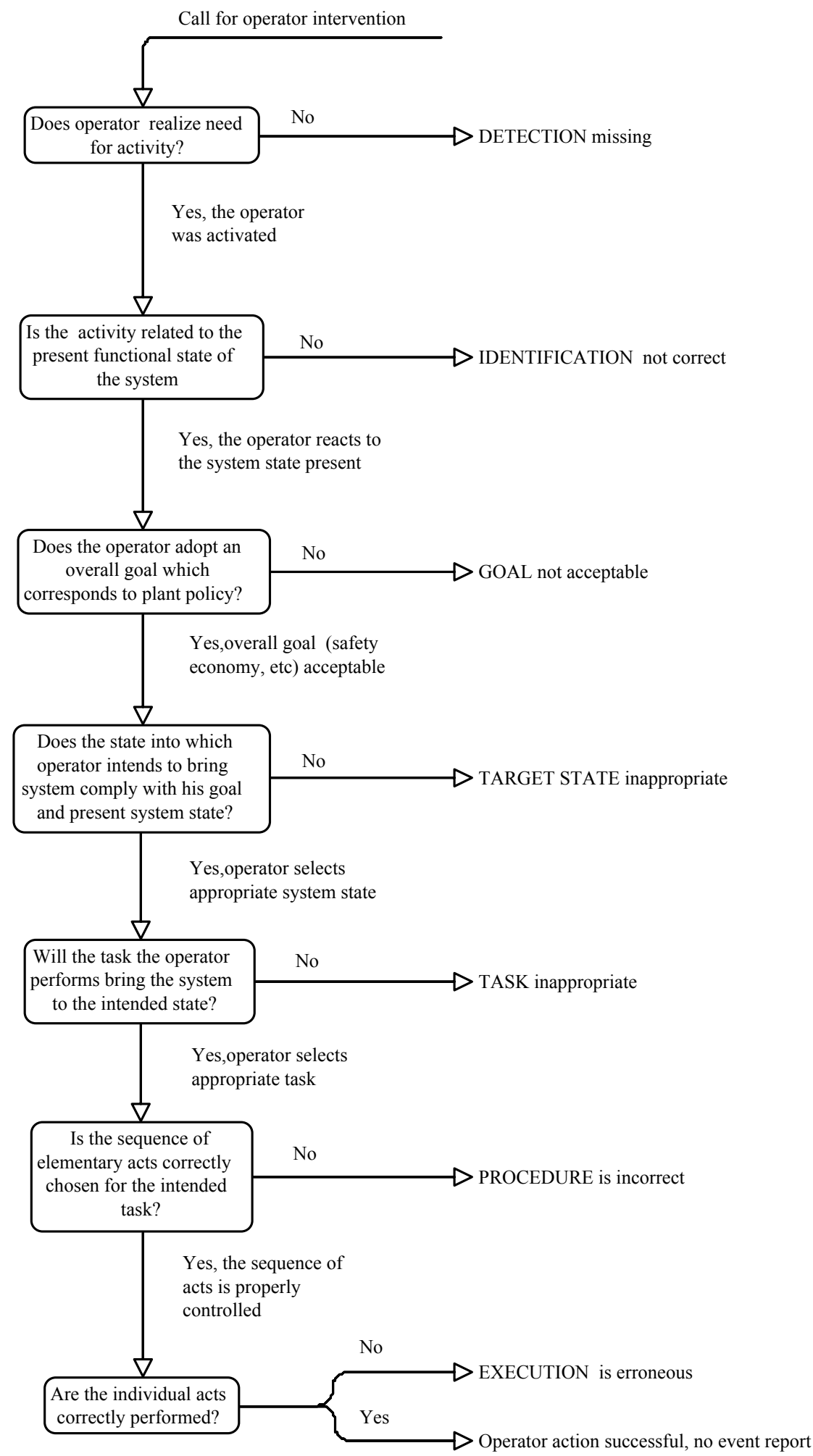

Figure 7. Guide to identification of the internal human malfunction from an event analysis. 
For cases including several inappropriate human decisions which are related in the chain of events, we normally only classify the first malfunction when the source of information is routine reports. This is due to the consideration that the situation following an erroneous decision is too complex to allow the analyst to judge the basis of the subsequent decisions and the normal classification categories may not apply. The variability, e.g., for human decision making, in a situation created by acts based on mis-identification of the state of the system, is only accessible through very detailed in situ analyses based on interviews.

A systematic guide to the analysis of simple routine event reports, to identify "what was wrong", is proposed in fig. 7.

\section{Mechanisms of Human Malfunction}

The categories of mechanisms of human malfunction are closely related to the categories of human behavior which are represented in the model of fig. 3. The categories of "internal human malfunction" and those of "mechanisms of human malfunctions", which are related to categories of internal human information processes and of internal human mechanisms, respectively, are basically different concepts and should therefore be considered separately during event analysis. During normal work situations, there is a rather close correlation between information process types and of mechanisms used for the activity. Since, however, event analysis will include situations of all degrees of familiarity for operators, we maintain that the categories of information processes and psychological mechanisms should be kept separate during analysis .

As discussed above, the mechanisms of malfunction must be deduced from the observable evidence by means of a model of human performance, and the analysis must therefore follow guidelines derived from such a model as, for instance, proposed derived from the three level model of fig. 3. The categories proposed should not be taken as a final set; it includes the categories which have been found typical from a preliminary analysis of 200 U.S. Licensee Event Reports (Rasmussen 1980). Since they have been found to cover the larger part of the cases, an immediate classification as proposed during event recording will save the effort for detailed data collection in the more complex situations.

An important situation for which detailed data collection and analysis are needed, is when operators respond to abnormal situations and have realized that knowledge-based reasoning is needed. In this knowledgebased domain there is very little correlation between the activity types of identification, decision and planning, and the underlying types of psychological mechanisms related to functional, causal deduction and search which will be applied in all the activities. In the present 
taxonomy, all mechanisms related to this level of behavior are lumped in the category of malfunction during inference such as inadequate consideration of causal conditions or side effects. Future studies, e.g. in training simulator sessions, will hopefully serve to make this category more detailed, as well as more infrequent categories now lumped in the category "other". It is therefore important to have good, free text descriptions of cases relating to these two categories.

\section{MECHANisms OF Human MALFUnCTION: How Did IT FAIL?}

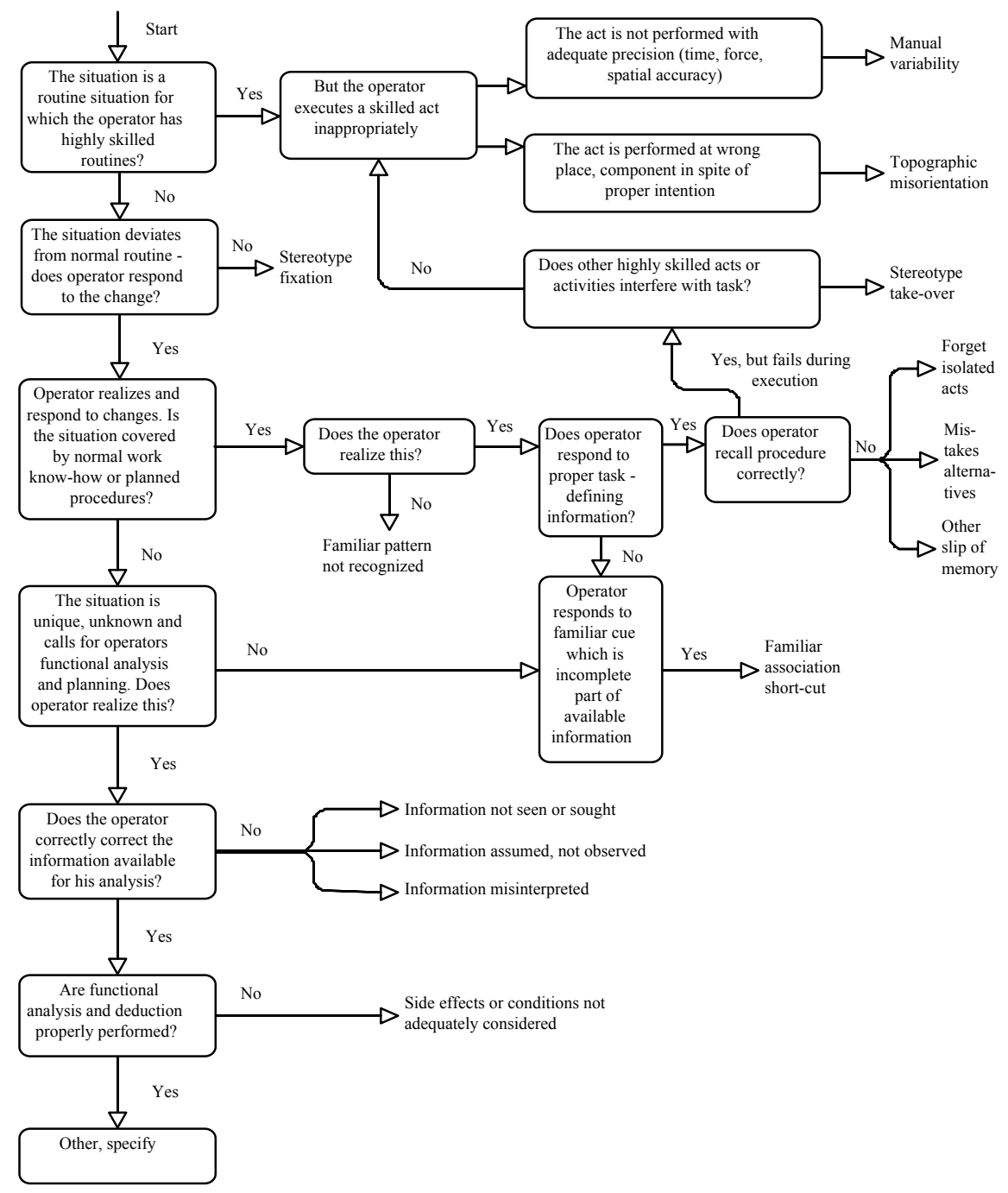

Figure 8. Guide for event analysis for identification of the psychological mechanisms leading to malfunction. 
CAUSES OF HUMAN MALFUNCTION: WHY DID IT FAIL?

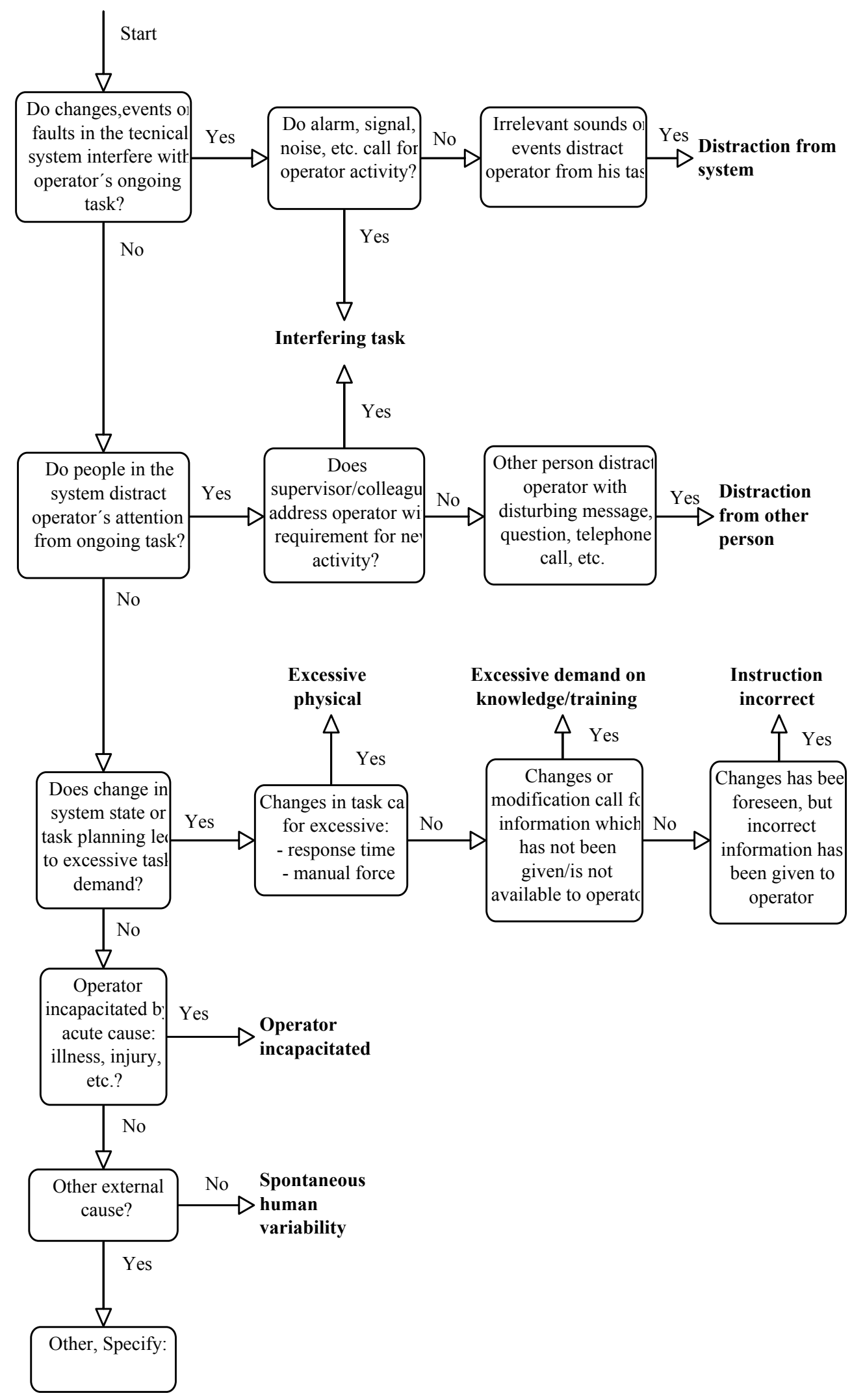

Figure 9. Guide for event analysis to identify external causes of human malfunction 


\section{Causes of Human Malfunction}

This category should identify the possible external causes of the inappropriate human action. As discussed in a previous section, a malfunction implies a change from normal or expected function and this change can be due to a spontaneous internal human variability or a change in the external task condition. Identification of possible external causes is important for several reasons. First of all, there is a natural tendency, when analyzing the chain of events implied in mal-operation of a system, to accept a human error as the explanation if an inappropriate human act is met by the causal backtracking "it is human to err". Special care is therefore needed to identify external causes. Secondly, such external causes may be important since they influence frequency and also may indicate causal coupling to other chains of event.

The category of causes within the present taxonomy should only be taken as illustrative. Specific sets should be identified in the different specific applications since they will be very context dependent. A decision tree to guide data collection can therefore only be a framework ensuring consideration of the major classes, such as the one illustrated in fig. 9 .

\section{Performance Shaping and Situation Factors}

These categories include general conditions which may influence error probability, but according to our definition do not cause errors. The distinction between the two categories is only caused by the fact that the set here called performance shaping factors can only be identified by careful human factors analysis, whereas the situation factors are readily recognizable. The class "task characteristics" is important in relation to error mechanisms, since it should identify whether the task is familiar to the person or not, and whether it is performed according to schedule or not. This information gives clue to identification of the level of behavior called upon (compare fig. 3).

\section{HUMAN ERROR PREDICTION}

In the sections above, references have been made to the use of the taxonomy for event analysis and some of the problems involved have been discussed. Event analysis implies a breakdown of the event into the features given by the taxonomy, and the quality of the taxonomy is related to the extent to which the causal flow and the mechanisms controlling the event propagation are maintained and can be regenerated from the data. When used for error prediction, the taxonomy must serve a synthesis of the relevant, possible chains of events during human 
performance from the elements contained in the categories together with an estimate of the probability or at least a ranking of the significance of the possible events. A discussion of the problems involved in prediction of human errors in general is presented elsewhere (Rasmussen 1979) and is outside the scope of the present paper and, accordingly, only an illustrative indication of the use of the taxonomy is considered.

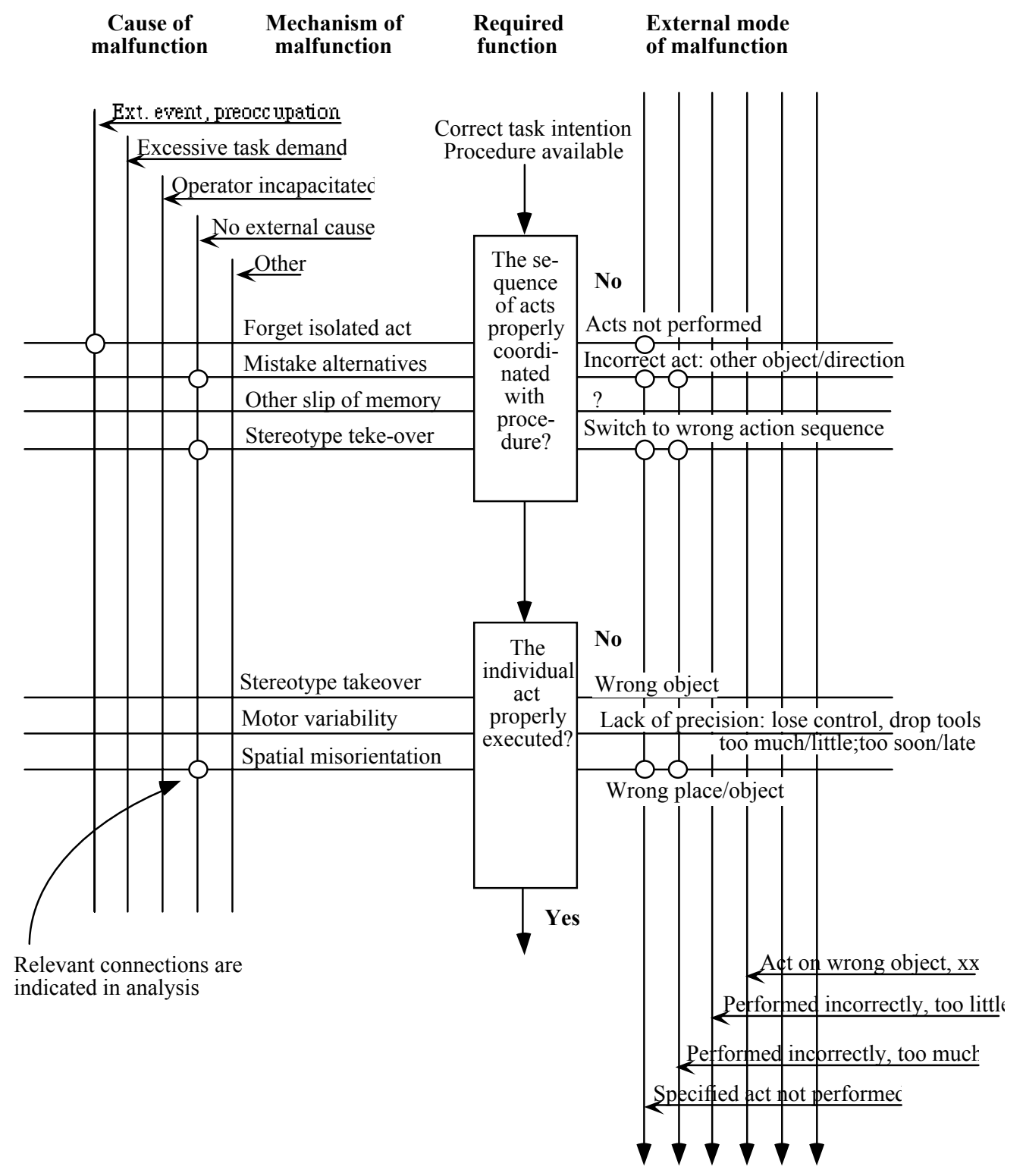

Figure 4. The figure illustrates how a error-mode-and-effect analysis tracing the causal chain through the dimensions of the error taxonomy can serve a detailed identification of possible extraneous effects of errors in cases where a reliable representation is available of the work procedure. This is, in particular, the case for operation of technical equipment. 
Prediction of human malfunction in a task considered in isolation is not very meaningful. The basis for any human error prediction in the present context will be the results of a functional analysis of the technical system or the task environment including a failure analysis. This analysis will serve to identify the requirements for human actions. It will specify the required human task in terms of an action sequence required to bring the system from one normal or disturbed state to another. At the same time, probability estimates for the relevant equipment failures and other non-human caused events will be very useful to serve as stop rules to prevent search for irrelevant human error mechanisms. When the task requirements related to different plant states are formulated, the problem of prediction is to determine whether the person will detect the need for action, identify the actual state of the system, choose the proper target state and so forth in short, the internal mental functions which are required by the task and could be wrong should be determined. Next, the internal mechanisms of malfunction are correlated to the required mental functions and their effect upon the actual task performance can be determined in detail. This means that the relevant external modes of failure for the actual task are identified and the related specific fault trees can be constructed. If we consider as a simple example the act of closing a valve, this can be unsuccessful due to different causal mechanisms. It may be opened fully instead of closed due to a "mistake of alternatives" or due to "stereotype fixation" (if it operates in reverse to usual). Closing may be omitted due to simple "slip of memory" with high probability, if the act is "functionally isolated" from the main course of the task. Or a wrong valve may be closed, in which case we have two coupled errors and it is important to predict the mistaken valve. Depending upon the error mechanism involved, this valve will be topographically close ("topographic mis-orientation"), have a name or label which can be mistaken ("mistake of alternatives", A for B for instance) or be part of a very familiar routine which is similar to the present task (psychologically close, "stereotype take-over"). The message of this simple example is that the causal relationships among mechanisms, mental function and task elements must be maintained during the analysis in order to identify the external mode of error and to relate frequencies or probabilities to the ultimate effects and to predict couplings between multiple errors. When the ultimate effect of the errors and potential for error correction are identified from the systems analysis, the probable causes of errors are estimated to judge error probability and potential for coupling to other events. Based on the proposed multi-facet taxonomy, general formats to guide identification of relevant chains of events including human error in systems analysis can be formed. An illustra- 
tive example for action errors is shown in figure 10, based on a combination of the categories of internal malfunction, mechanisms of malfunction, together with causes and external effects. A practical implementation of such pre-formatted analysis guides have been developed by Taylor (1979). Since errors of intention are very complex and situation specific, such general guides for this kind of error are not feasible at present. However, error prediction for familiar tasks for which the procedure is known is an important part of systems reliability analysis and risk analysis and tools like the action analysis format of fig. 10 have proved useful.

\section{CONCLUSION}

In the present paper, it has been stressed several times that the important aspect of the proposed taxonomy is the structure, not the elements used within the various categories. There is, at present, a widespread interest in quantification of human performance which appears to be somewhat premature, since the qualitative structures and categories which are necessary to define the items to be measured or counted are not properly sorted out. This cannot be done without careful studies of human performance and errors in actual work situations in order to reach a better understanding of the complexity of human error situations and the data needed to characterize them. The purpose of the present taxonomy has been to contribute to the basis for such studies by means of more systematic experiments in data collection schemes for real-life work situations.

\section{REFERENCES}

Fitts, P. M. and Jones, R. E., 1947, "Analysis of Factors Contributing to 460 "PilotError" Experiences in Operating Aircraft Controls", Report TSEAA-694-12 Aeromedical Laboratory, Wright-Patterson Air Force Base, Dayton, Ohio, reprinted in: Selected Papers on Human Factors in the Design and Use of Control Systems, Sinaiko, W. H. (Ed.), New York, Dover, 1961.

Hollnagel, E., Pedersen, O.M. and Rasmussen, J., 1981, "Notes on Human Performance Analysis", Risø-M-2285.

Moray, N., 1981, "The Role of Attention in the Detection of Errors and the Diagnosis of Failures in Man-Machine Systems", to be published in: Human Detection and Diagnosis of System Failures, J. Rasmussen and W. B. Rouse (Eds.), New York: Plenum Press.

Norman, D. A., 1979, "Slips of the Mind and an Outline for a Theory of Action", Report CHIP 88, Center for Human Information Processing, University of California, San Diego.

Norman, D. A., 1980, "Errors in Human Performance", Report No. 8004, Center for Human Information Processing, University of California, San Diego.

Rasmussen, J., 1976, "Outlines of a Hybrid Model of the Process Operator", in: Monitoring Behaviour and Supervisory Control, Sheridan and Johannsen (Eds.), Plenum Press, New York. 
Rasmussen, J., 1979, "Notes on Human Error Analysis and Prediction", in: Synthesis and Analysis Methods for Safety and Reliability Studies, Apostolakis, G., and Volta, G. (Eds.), Plenum Press, London.

Rasmussen, J., 1980, "What Can Be Learned from Human Error Reports?", in: Changes in Working Life, Duncan, K. D., Gruneberg, M. M., and Wallis, D. (Eds.), John Wiley \& Sons.

Rasmussen, J., 1980, "Some Trends in Man-Machine Interface Design for Industrial Process Plants", Risø-M-2228, also in proceedings of "ASSOPO 80" an IFIP/IFAC Symposium, Norway.

Rasmussen, J ., 1981, "Models of Mental Strategies in Process Plant Diagnosis", to be published in: Human Detection and Diagnosis of System Failures, J. Rasmussen and W. B. Rouse (Eds.), New York: Plenum Press.

Rasmussen, J. and Pedersen, O. M. P.; Carnino, A. and Griffon, M .; Mancini, G.; Gagnolet, A., 1981, "Classification System for Reporting Events Involving Human Malfunction", Risø-M-2240 .

Reason, J. T., 1975, "How did I Come to Do That?", New Behaviour, April 24, 1975.

Reason, J . T ., 1976, "Absent Minds", New Society, November 4, 1976.

Reason, J. T., 1977, "Skill and Error in Everyday Life", in: Adult Learning, M. Howe (Ed.), London: Wiley.

Swain, A., 1976, "Sandia Human Factors Program for Weapon Development", SAND 76-0326 Sandia Laboratories.

Swain, A., 1980, "Handbook of Human Reliability Analysis with Emphasis on NPP Applications", Draft Report NUREG/CR-1 278 .

Taylor, J. R., 1979, "A Background to Risk Analysis", Vol. 1 to 4, Electronics Department, Risø National Laboratory. 